\title{
Composição de espécies de Arctiidae (Insecta, Lepidoptera) em áreas de Cerrado
}

\author{
Viviane G. Ferro' ${ }^{1} \&$ Ivone R. Diniz ${ }^{2}$ \\ ${ }^{1}$ Departamento de Zoologia, Laboratório de Ecologia de Insetos, Instituto de Biociências, Universidade Federal do Rio Grande \\ do Sul. Avenida Bento Gonçalves 9500, Bloco IV, Prédio 43435, sala 227, 91501-970 Porto Alegre, Rio Grande do Sul, \\ Brasil. E-mail: vgferro@yahoo.com \\ ${ }_{2}^{2}$ Departamento de Zoologia, Instituto de Ciências Biológicas, Universidade de Brasília. 70910-900 Brasília, Distrito Federal, \\ Brasil.E-mail: irdiniz@unb.br
}

\begin{abstract}
Composition of the Arctiidae species (Insecta, Lepidoptera) in Cerrado areas. The Cerrado biome covers $23 \%$ of the Brazilian territory and includes nearly one third of the estimated biota of the country. The aim of this article is to know the species composition of the nocturnal Arctiidae and describe the betadiversity patterns among 14 Cerrado areas. We collected a total of 1,016 individuals, representing 197 morphospecies. Estimates of species richness using Jackknife 2 and Chao 2 indicated that the region should contain, respectively, 375.7 and 383.8 species. The regression between species richness and number of vegetation types in the sampled areas was not significant. The beta-diversity (Sorensen distance) among study sites was positively related to geographical distances. The multivariate analyses indicated that, except for Pedregulho, study areas in São Paulo State were distinct from those in Goiás and Tocantins States. Pooling study sites within vegetation types revealed that fauna of gallery forests and "cerradões" were similar and, together, were different from those collected in open vegetacional formations ("campo sujo" and "cerrado sensu stricto"). We suggested that vegetation characteristics and geographical distance are important factors in the determination of Arctiidae assemblages in the Brazilian Cerrado.

KEY WORDS. Beta-diversity; Cerrado vegetation types; geographical distances; species richness.
\end{abstract}

RESUMO. O bioma Cerrado ocupa $23 \%$ do território brasileiro e abriga aproximadamente um terço da biota estimada para o país. $\mathrm{O}$ objetivo deste trabalho é conhecer a composição de espécies de Arctiidae noturnos e descrever os padrões de diversidade beta entre 14 áreas de Cerrado. Foram coletados 1.016 indivíduos, representando 197 morfo-espécies. Estimativas de riqueza utilizando Jackknife 2 e Chao 2 indicaram que a região deve conter, respectivamente, 375,7 e 383,8 espécies. A regressão entre a riqueza de espécies observada e o número de fitofisionomias existente nas áreas amostradas não foi significativa. A diversidade beta (distância de Sorensen) entre as localidades estudadas foi positivamente relacionada com as distâncias geográficas. A análise multivariada indicou que, com exceção de Pedregulho, as áreas do estado de São Paulo foram distintas daquelas dos estados de Goiás e Tocantins. Agrupando os sítios por fitofisionomia, foi verificado que a fauna das matas de galeria e dos cerradões foram similares e formaram um grupo distinto daquele composto por formações abertas (campo sujo e cerrado sentido restrito). Sugere-se que os tipos de vegetação e as distâncias geográficas são fatores importantes na determinação das assembléias de Arctiidae no Cerrado brasileiro.

PALAVRAS-CHAVE. Diversidade beta; distâncias geográficas; fitofisionomias de cerrado; riqueza de espécies.

O bioma Cerrado ocupa 23\% do território do Brasil, sendo excedido em área apenas pela Amazônia (RATTER et al. 1997). A vegetação do Cerrado é caracterizada por fitofisionomias florestais, savânicas e campestres, em função de um gradiente decrescente na densidade de árvores. O cerradão e as matas de galeria, ciliar e seca correspondem às fitofisionomias florestais. As fitofisionomias savânicas são compostas pelo cerrado sentido restrito, o parque cerrado, o palmeiral e a vereda. Já as fitofisionomias campestres são os campos limpo, sujo e rupestre (Ribeiro \& Walter 1998).
A região do Cerrado abriga aproximadamente um terço da biota brasileira, com riqueza mínima estimada de $320 \mathrm{mil}$ espécies (DiAs 1996). A flora vascular do Cerrado é a mais rica de todas as savanas neotropicais, com cerca de 10 mil espécies (RATter et al. 1997). Entre os animais, existem 837 espécies de aves, 161 de mamíferos, 150 de anfíbios, 120 de répteis (Myers et al. 2000) e há uma estimativa de 90 mil espécies de insetos (Dias 1996). Brown Jr \& Mielke $(1967,1968)$ e Mielke (1968) estimaram mais de 900 espécies de borboletas para o planalto central.

Revista Brasileira de Zoologia 24 (3): 635-646, setembro 2007 
O Cerrado está incluído entre os 25 "hotspots" mundiais, isto é, áreas com grande endemismo e menos de 30\% de vegetação original remanescente (Myers et al. 2000). As plantas vasculares do Cerrado possuem as maiores taxas de endemismo (44\%), seguida das taxas dos anfíbios (30\%), répteis (20\%) (Myers et al. 2000), aves (11\%) (MACEDo 2002) e mamíferos (9,3\%) (MARINHOFilHo et al. 2002). Entre os invertebrados, 19\% dos lepidópteros são endêmicos da região do Cerrado, sendo que essa proporção varia consideravelmente entre as famílias de borboletas (de 9\% para os Pieridae a 24\% para os Riodinidae) (BROWN JR \& GIFForD 2002). CAMARgo \& BeCKer (1999) encontraram uma taxa de endemismo de $12,6 \%$ para as mariposas Saturniidae do Cerrado.

Myers et al. (2000) estimaram que 80\% do Cerrado já foi alterado pelo homem. As regiões com maiores impactos antrópicos estão nos estados de Mato Grosso do Sul, Goiás, São Paulo, a fronteira de São Paulo com o Paraná e o Triângulo Mineiro (Mantovani \& Pereira 1998). As unidades de conservação federais e estaduais cobrem apenas 1,5\% da área do Cerrado (DiAs 1996). Sabe-se que, pelo menos, três espécies de anfíbios, 15 de répteis e 33 de aves estão ameaçadas de extinção no bioma (Conservation International et al. 1999).

Em decorrência das altas taxas de conversão do Cerrado em áreas destinadas à agropecuária ou outras formas de ocupação humana, faz-se cada vez mais necessário o conhecimento da diversidade biológica para que se possa entender o funcionamento das comunidades e desenvolver planos de manejo para sua preservação. Considerando que mais da metade das espécies descritas no mundo são insetos, este grupo é de fundamental importância para o entendimento dos padrões da biodiversidade.

Borboletas e mariposas são muito utilizadas em estudos sobre conservação de habitats degradados porque respondem rapidamente às mudanças na vegetação e no clima, formam um grupo altamente abundante e diverso, sua taxonomia é relativamente bem conhecida e as técnicas de coleta são simples (DeVries et al. 1997). Segundo Hilty \& Merenlender (2000), as famílias Arctiidae, Saturniidae e Sphingidae estão entre os lepidópteros noturnos mais utilizados como bioindicadores no monitoramento de ecossistemas.

A família Arctiidae compreende cerca de 11 mil espécies de mariposas de tamanho pequeno a médio e é distribuída por todo o mundo (Scoble 1995). Ocorrem cerca de seis mil espécies na Região Neotropical (HeppNer 1991) e há uma estimativa de duas mil espécies para o Brasil (Brown Jr \& Freitas 1999). Segundo Jacobson \& Weller (2002), essas mariposas estão divididas nas subfamílias Arctiinae, Lithosiinae e Syntominae, sendo que a última não ocorre nas Américas. Este trabalho tem como objetivos examinar a composição das espécies noturnas de Arctiidae em 14 áreas de Cerrado e analisar se existe relação entre o número de fitofisionomias e a riqueza de espécies desses lepidópteros. Além disso, pretende-se verificar se existe relação entre a diversidade beta e a distância geográfica entre essas áreas. São discutidos possíveis parâmetros que poderiam explicar os padrões de diversidade beta encontrados.

\section{MATERIAL E MÉTODOS}

As coletas noturnas foram feitas em localidades de Cerrado nos estados de Goiás (GO), São Paulo (SP) e Tocantins (TO) (Fig. 1, Tab. I). A escolha das localidades dependeu da intensidade de coletas prévias de Arctiidae na região do bioma, priorizando-se localidades pouco representadas ou sem registro em coleções.

Em cada localidade foram feitas amostragens rápidas (de uma a cinco noites) durante a estação chuvosa (novembro a março) nos anos de 2004 e 2005 . As coletas foram feitas excluindo o período entre os cinco dias antes e cinco dias após a primeira noite de lua cheia, período em que há maior luminosidade da lua nas primeiras horas da noite. Os adultos noturnos foram coletados através de duas fontes luminosas (lâmpadas mistas de mercúrio de $250 \mathrm{~W}$ ) suspensas a 1,6m do solo, cada uma refletida em um tecido branco estendido na vertical. As lâmpadas foram dispostas em pontos separados por cerca de 100m e permaneceram acesas durante três horas a partir do anoitecer. O horário do anoitecer variou em torno de 30 minutos entre as áreas, em virtude da diferença de latitude e da densidade da vegetação.

As coordenadas das localidades de coleta foram adquiridas através de GPS. Também foram registrados os tipos de fitofisionomias presentes (campo, cerrado sentido restrito, cerradão ou mata de galeria) e o estado de preservação da vegetação em cada uma das localidades. No contexto deste trabalho, a palavra alterada refere-se à invasão ou reflorestamento de pinheiro (Pinus spp) e eucalipto, presença de gado e/ou retirada de vegetação original.

Os Arctiidae foram capturados e mortos com auxílio de frascos mortíferos. No dia seguinte, ainda no campo, os indivíduos foram montados e secos em estufa. Posteriormente, estes foram identificados por comparação com a Coleção Becker (VOB), cujas espécies foram comparadas com os tipos. As identificações foram confirmadas pelo Dr. Vítor Becker. Os exemplares testemunha estão depositados na Coleção Entomológica do Departamento de Zoologia da Universidade de Brasília.

A relação entre a riqueza de espécies observada e o número de fitofisionomias nas localidades foi avaliada através de regressão linear. Foi feito também o teste de mantel para verificar se havia relação entre a diversidade beta (medida como distância de Sorensen, com dados de presença e ausência) e a distância geográfica $(\mathrm{km})$ entre as localidades. A regressão linear e o teste de mantel (incluindo os cálculos das matrizes de distância de Sorensen e geográfica) foram realizados no programa $\mathrm{R}$ (R Development Core Team 2004).

Estimativas de riqueza de espécies para a região das localidades amostradas foram feitas usando os estimadores não paramétricos Jackknife de segunda ordem $\left[S^{*}=S_{o b s}+\frac{L(2 n-3)}{n}-\frac{M(n-2)^{2}}{n(n-1)}\right]$ e Chao $2\left[S^{*}=S_{o b s}+\frac{L^{2}}{2 M}\right]$, onde $S^{\star}$ é a riqueza de espécies extrapolada pelo índice, $S_{o b s}$ é a riqueza de espécies observada no estudo, $L$ é o número de espé- 

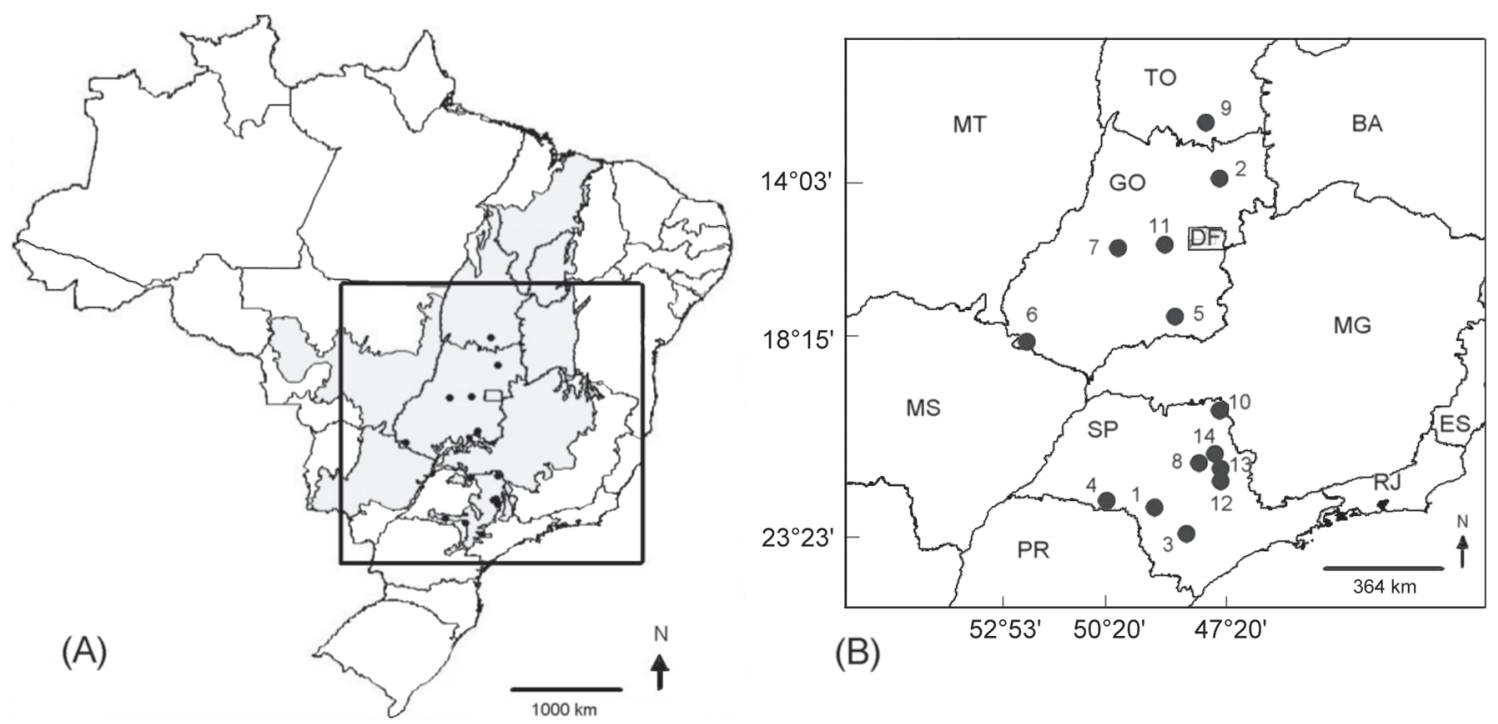

(B)

Figura 1. "(A) Mapa do Brasil mostrando a região nuclear do Cerrado (em cinza) e (B) detalhe da região delimitada pelo quadrado, indicando a posição das localidades onde foram realizadas as coletas. (1) Águas de Santa Bárbara, (2) Alto Paraíso de Goiás, (3) Angatuba, (4) Assis, (5) Caldas Novas, (6) Chapadão do Céu, (7) Goiás, (8) Luis Antônio, (9) Paranã, (10) Pedregulho, (11) Pirenópolis, (12) Porto Ferreira, (13) Santa Rita do Passa Quatro e (14) São Simão.

Tabela. I. Estado, coordenadas geográficas, datas em que as coletas foram realizadas, fitofisionomias de cerrado amostradas e estado de conservação da vegetação nas 14 localidades estudadas. As informações dentro dos parênteses indicam as unidades de conservação onde as coletas foram realizadas e a ausência de parênteses indica coleta em área particular. A fitofisionomia cerrado refere-se ao cerrado sentido restrito e a fitofisionomia mata refere-se à mata de galeria.

\begin{tabular}{|c|c|c|c|c|c|c|}
\hline Código & Localidades & Estado & $\begin{array}{c}\text { Coordenadas } \\
(\mathrm{S}, \mathrm{W})\end{array}$ & Datas das coletas & Fitofisionomias & Estado de conservação \\
\hline 1 & $\begin{array}{l}\text { Águas de Santa Bárbara (Estação } \\
\text { Ecológica de Santa Bárbara) }\end{array}$ & SP & $22^{\circ} 48^{\prime}, 49^{\circ} 13^{\prime}$ & $3 / 11 / 2005$ & Cerrado, mata & Alterado (gado e pinus) \\
\hline 2 & $\begin{array}{l}\text { Alto Paraíso de Goiás (Parque Nacional } \\
\text { da Chapada dos Veadeiros) }\end{array}$ & GO & $14^{\circ} 03^{\prime}, 47^{\circ} 30^{\prime}$ & $24-25 / I I I / 2004$ & Cerrado & Preservado \\
\hline 3 & $\begin{array}{l}\text { Angatuba (Estação Ecológica de } \\
\text { Angatuba) }\end{array}$ & SP & $23^{\circ} 23^{\prime}, 48^{\circ} 19^{\prime}$ & $\begin{array}{l}19 / 11 / 04 \text { e } 29- \\
30 / I / 2005\end{array}$ & Cerradão & Preservado* \\
\hline 4 & Assis (Estação Ecológica de Assis) & SP & $22^{\circ} 36^{\prime}, 50^{\circ} 22^{\prime}$ & $31 / I-2 / I I / 2005$ & Cerradão, mata & Preservado* \\
\hline 5 & $\begin{array}{l}\text { Caldas Novas (Parque Estadual da Serra } \\
\text { de Caldas Novas) }\end{array}$ & GO & $17^{\circ} 43^{\prime}, 48^{\circ} 36^{\prime}$ & $22-23 / \mathrm{III} / 2004$ & Cerrado & Preservado \\
\hline 6 & $\begin{array}{l}\text { Chapadão do Céu (Parque Nacional de } \\
\text { Emas) }\end{array}$ & GO & $18^{\circ} 15^{\prime}, 52^{\circ} 53^{\prime}$ & $6-10 / 11 / 2005$ & $\begin{array}{l}\text { Campo sujo, } \\
\text { cerrado, mata }\end{array}$ & Preservado \\
\hline 7 & Goiás & GO & $15^{\circ} 39^{\prime}, 50^{\circ} 12^{\prime}$ & $23 / 111 / 2004$ & Cerrado & Alterado (gado) \\
\hline 8 & Luis Antônio (Estação Ecológica de Jataî) & SP & $21^{\circ} 36^{\prime}, 47^{\circ} 47^{\prime}$ & $9 / 11 / 2004$ & Cerradão & Preservado \\
\hline 9 & Paranã & TO & $12^{\circ} 54^{\prime}, 47^{\circ} 37^{\prime}$ & $11-12 / I I I / 2004$ & Cerrado & Alterado (gado) \\
\hline 10 & $\begin{array}{l}\text { Pedregulho (Parque Estadual das Furnas } \\
\text { do Bom Jesus) }\end{array}$ & SP & $20^{\circ} 14^{\prime}, 47^{\circ} 27^{\prime}$ & $12-13 / 11 / 2005$ & $\begin{array}{l}\text { Campo sujo, } \\
\text { mata }\end{array}$ & Alterado (gado) \\
\hline 11 & Pirenópolis & GO & $15^{\circ} 48^{\prime}, 49^{\circ} 00^{\prime}$ & $26 / 111 / 2004$ & Mata & Alterado (turismo) \\
\hline 12 & $\begin{array}{l}\text { Porto Ferreira (Parque Estadual de Porto } \\
\text { Ferreira) }\end{array}$ & SP & $21^{\circ} 51^{\prime}, 47^{\circ} 24^{\prime}$ & $12 / 11 / 2004$ & Cerradão, mata & Preservado \\
\hline 13 & $\begin{array}{l}\text { Santa Rita do Passa Quatro (Parque } \\
\text { Estadual de Vassununga) }\end{array}$ & SP & $21^{\circ} 36^{\prime}, 47^{\circ} 37^{\prime}$ & $11 / 11 / 2004$ & Cerradão & Preservado \\
\hline 14 & São Simão & SP & $21^{\circ} 26^{\prime}, 47^{\circ} 24^{\prime}$ & $10 / I I / 2004$ & Mata & Alterado (gado) \\
\hline
\end{tabular}

* Apesar de existir plantio de Pinus spp. na unidade de conservação, a região do ponto de coleta era bem preservado. 
cies que ocorreu em apenas uma localidade, $n$ é o número de localidades amostradas e $M$ é o número de espécies que ocorreu em duas localidades. Os cálculos foram feitos com auxílio de calculadora. Segundo Colwell \& Coddington (1994) estes dois estimadores fornecem as estimativas com maior acuidade e menor viés para conjunto de dados com pequeno número de amostras.

Com o propósito de obter uma melhor caracterização das semelhanças entre as faunas das localidades estudadas, foram feitas análises de classificação aglomerativa (Unweighted PairGroups Method using Arithmetic Averages - UPGMA) combinando a fauna por localidade e, também, por fitofisionomia (campo, cerrado sentido restrito, cerradão e mata de galeria). Estas análises foram feitas com o auxílio do programa PCORD versão 4.17 (McCune \& MEFFord 1997).

\section{RESULTADOS}

Foram coletados 1016 arctiídeos de duas subfamílias. Ao todo, foram amostradas 197 espécies, sendo 159 nominadas, 17 identificadas apenas pelo gênero e 21 não identificadas. $\mathrm{O}$ número de indivíduos e de espécies coletados em cada localidade encontra-se na tabela II e a lista das espécies no apêndice I. A subfamília Arctiinae apresentou o maior número de espécies (132). Seis tribos de Arctiinae (Arctiini, Callimorphini, Ctenuchini, Euchromiini, Pericopini e Phaegopterini) e três de Lithosiinae (Cisthenini, Eudesmiini e Lithosiini) estiveram presentes nas coletas (Apêndice I).

Estimou-se, através do Jackknife de segunda ordem, uma riqueza de 375,7 espécies para a região das 14 localidades. Desse modo, foram coletados em 26 noites, 52,4\% da fauna de Arctiidae estimada pelo índice. $\mathrm{O}$ valor do estimador Chao 2 foi um pou- co maior, mas bastante próximo, com 383,8 espécies.

Nenhuma espécie ocorreu em todas as localidades, sendo a maioria $(58,8 \%)$ encontrada em apenas uma localidade (Tab. II). As localidades de Goiás, Chapadão do Céu, Angatuba e Alto Paraíso de Goiás foram aquelas que apresentaram as maiores porcentagens de espécies únicas (Tab. II). As espécies mais comumente encontradas foram os arctiíneos Aclytia heber (Cramer, 1780), Dycladia lucetius (Stoll, 1781) (presentes em 12 localidades), Lepidoneiva erubescens (Butler, 1876) (em 10) e os litosiíneos Euthyone simplex (Walker, 1854), Nodozana jucunda Jones, 1914 e Odozana domina (Schaus, 1896) (presentes em nove localidades). Os gêneros com maior número de espécies coletadas foram Cosmosoma Hübner, [1823] (Arctiinae) (com 11 espécies), Cisthene Walker, 1854 (Lithosiinae) (com nove) e Agylla Walker, 1854 (Lithosiinae) (com oito) (Apêndice 1). A abundância das espécies variou de um a 58 indivíduos, sendo que aquelas espécies representadas por apenas um indivíduo representaram 41,6\% do total das espécies coletadas (Tab. II).

A regressão entre o número de fitofisionomias nas localidades e a riqueza observada foi positiva, mas não significativa $\left(\mathrm{r}^{2}=0,2, \mathrm{p}=0,1, \mathrm{n}=14\right)$. $\mathrm{O}$ teste de mantel entre a diversidade beta (medida como distância de Sorensen) e a distância geográfica entre as localidades foi positivo e fortemente significativo $\left(\mathrm{r}^{2}=0,4, \mathrm{p}<0,01, \mathrm{n}=91\right)$. As localidades de Águas de Santa Bárbara e Assis e de Luis Antônio e Santa Rita do Passa Quatro (todas em SP) apresentaram a fauna mais semelhante entre si (índice de similaridade de Sorensen igual a 0,77 para ambos os pares) e de Chapadão do Céu e Goiás (GO) a mais distinta $(0,07)$. Os índices de similaridade de Sorensen entre todas as localidades estudadas estão na tabela III.

Tabela. II. Número de indivíduos e de morfo-espécies, porcentagem de morfo-espécies exclusivas e de morfo-espécies que ocorreram com apenas um indivíduo em cada uma das localidades amostradas.

\begin{tabular}{|c|c|c|c|c|}
\hline Localidades & $\begin{array}{l}\text { Indivíduos } \\
\text { (n) }\end{array}$ & $\begin{array}{l}\text { Morfo-espécies } \\
\text { (n) }\end{array}$ & $\begin{array}{l}\text { Morfo-espécies } \\
\text { exclusivas (\%) }\end{array}$ & $\begin{array}{c}\text { Morfo-espécies com apenas } \\
\text { um indivíduo (\%) }\end{array}$ \\
\hline Águas de Santa Bárbara & 51 & 25 & 4,0 & 52,0 \\
\hline Alto Paraíso de Goiás & 38 & 25 & 40,0 & 72,0 \\
\hline Angatuba & 257 & 69 & 40,6 & 34,8 \\
\hline Assis & 160 & 43 & 16,3 & 37,2 \\
\hline Caldas Novas & 66 & 50 & 28,0 & 82,0 \\
\hline Chapadão do Céu & 174 & 44 & 40,9 & 43,2 \\
\hline Goiás & 17 & 14 & 42,8 & 78,6 \\
\hline Luis Antônio & 42 & 22 & 13,6 & 59,0 \\
\hline Paranã & 20 & 20 & 30,0 & 100,0 \\
\hline Pedregulho & 40 & 24 & 29,1 & 58,3 \\
\hline Pirenópolis & 18 & 17 & 29,4 & 94,1 \\
\hline Porto Ferreira & 48 & 22 & 4,5 & 45,4 \\
\hline Santa Rita do Passa Quatro & 45 & 21 & 9,5 & 33,3 \\
\hline São Simão & 40 & 26 & 30,7 & 76,9 \\
\hline Total & 1.016 & 197 & 58,8 & 41,6 \\
\hline
\end{tabular}

Revista Brasileira de Zoologia 24 (3): 635-646, setembro 2007 
Tabela. III. Índices de similaridade de Sorensen entre as 14 localidades de cerrado amostradas. Os números das localidades correspondem aos da tabela I.

\begin{tabular}{|c|c|c|c|c|c|c|c|c|c|c|c|c|c|}
\hline Localidades & 1 & 2 & 3 & 4 & 5 & 6 & 7 & 8 & 9 & 10 & 11 & 12 & 13 \\
\hline 2 & 0,53 & & & & & & & & & & & & \\
\hline 3 & 0,59 & 0,27 & & & & & & & & & & & \\
\hline 4 & 0,77 & 0,37 & 0,61 & & & & & & & & & & \\
\hline 5 & 0,49 & 0,50 & 044 & 0,48 & & & & & & & & & \\
\hline 6 & 0,45 & 0,36 & 0,41 & 0,44 & 0,54 & & & & & & & & \\
\hline 7 & 0,36 & 0,28 & 0,18 & 0,20 & 0,29 & 0,07 & & & & & & & \\
\hline 8 & 0,61 & 0,51 & 0,32 & 0,64 & 0,48 & 0,38 & 0,30 & & & & & & \\
\hline 9 & 0,39 & 0,52 & 0,29 & 0,24 & 0,53 & 0,39 & 0,32 & 0,34 & & & & & \\
\hline 10 & 0,29 & 0,30 & 0,31 & 0,46 & 0,43 & 0,22 & 0,38 & 0,39 & 0,47 & & & & \\
\hline 11 & 0,26 & 0,26 & 0,30 & 0,36 & 0,37 & 0,35 & 0,24 & 0,52 & 0,38 & 0,35 & & & \\
\hline 12 & 0,44 & 0,38 & 0,55 & 0,60 & 0,44 & 0,38 & 0,38 & 0,39 & 0,59 & 0,18 & 0,37 & & \\
\hline 13 & 0,52 & 0,39 & 0,47 & 0,53 & 0,60 & 0,39 & 0,41 & 0,77 & 0,58 & 0,47 & 0,61 & 0,62 & \\
\hline 14 & 0,47 & 0,47 & 0,34 & 0,49 & 0,33 & 0,26 & 0,19 & 0,66 & 0,24 & 0,22 & 0,18 & 0,18 & 0,62 \\
\hline
\end{tabular}

A análise de UPGMA para a fauna coletada nas diferentes localidades de Cerrado (Fig. 2) gerou dois grandes grupos: um formado por Goiás (GO) e Pedregulho (SP) e outro pelas demais localidades. Na segunda divisão, Pirenópolis (GO) difere das outras localidades. Na terceira divisão, as localidades de SP diferiram das localidades do Centro-Oeste. Na quarta divisão, as localidades de SP foram divididas em dois grupos: um formado por localidades do sudoeste (Águas de Santa Bárbara, Assis e Angatuba) e outro por localidades do nordeste do Estado (Luis Antônio, Santa Rita do Passa Quatro, Porto Ferreira e São Simão). Também na quarta divisão, as localidades de GO e TO foram divididas em um grupo do norte (Alto Paraíso de Goiás e Paranã) e outro do sul (Caldas Novas e Chapadão do Céu). As localidades de SP (exceto Pedregulho) apresentaram maior similaridade entre si do que as de GO e TO (Fig. 2).

A análise de UPGMA para a fauna coletada em diferentes fitofisionomias de Cerrado (Fig. 3) mostrou que a composição de Arctiidae em matas de galeria é muito semelhante à dos cerradões. Já a fauna de vegetação aberta (campo sujo) formou um grupo bem diferenciado das demais fitofisionomias.

\section{DISCUSSÃO}

A maioria das espécies $(58,8 \%)$ de Arctiidae foi encontrada em apenas uma localidade. Além disso, 41,6\% das espécies foram representadas por apenas um indivíduo. Este padrão de freqüência de ocorrência e de baixa abundância das espécies também foi observado em insetos herbívoros de florestas tropicais úmidas (Novotny \& BASSET 2000), assim como em adultos de mariposas (CAMARGo 1999) e larvas de Lepidoptera (MARQUis et al. 2002) do Cerrado. Segundo esses últimos autores, a baixa umidade do ar, o baixo conteúdo nutricional das plantas hospedeiras e a pressão de inimigos naturais poderiam explicar a baixa abundância de grande parte dos lepidópteros neste bioma.

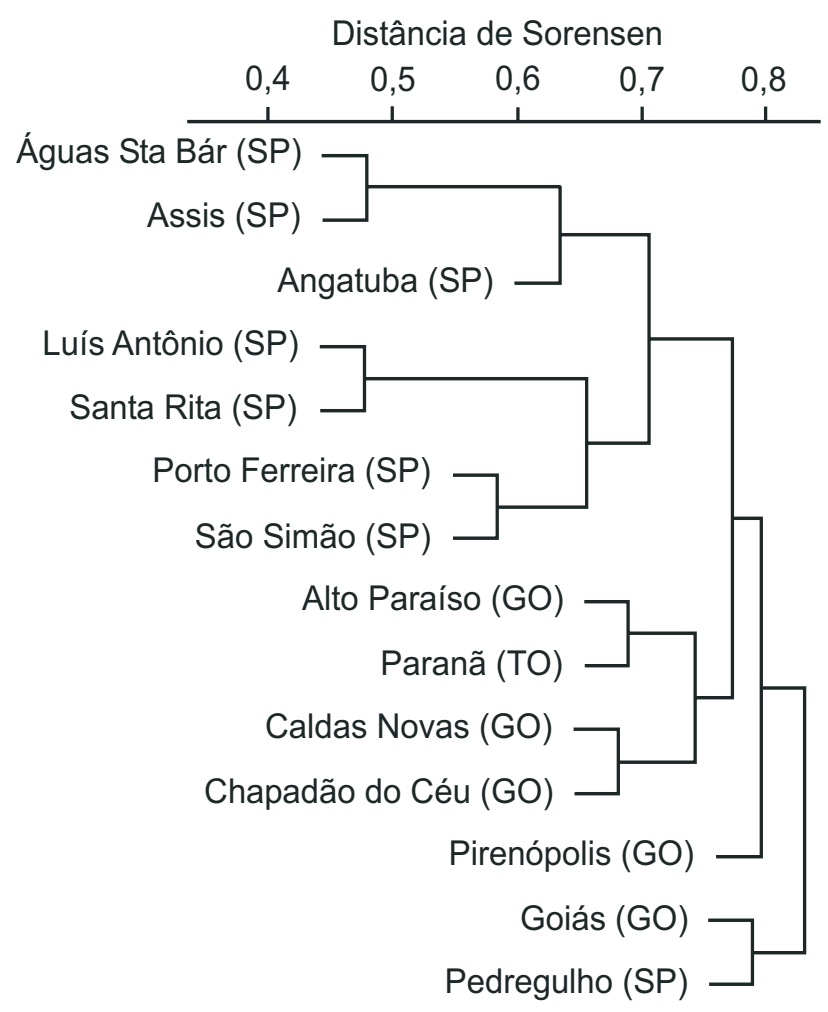

Figura 2. Classificação das 14 localidades de Cerrado por UPGMA, utilizando o índice de Sorensen como medida de distância.

Vários estudos têm sugerido que a diversidade florística é um dos fatores com maior influência na riqueza de espécies de Lepidoptera (Robinson \& Tuck 1993). Durigan et al. (2003), por exemplo, encontraram uma relação positiva entre o núme- 


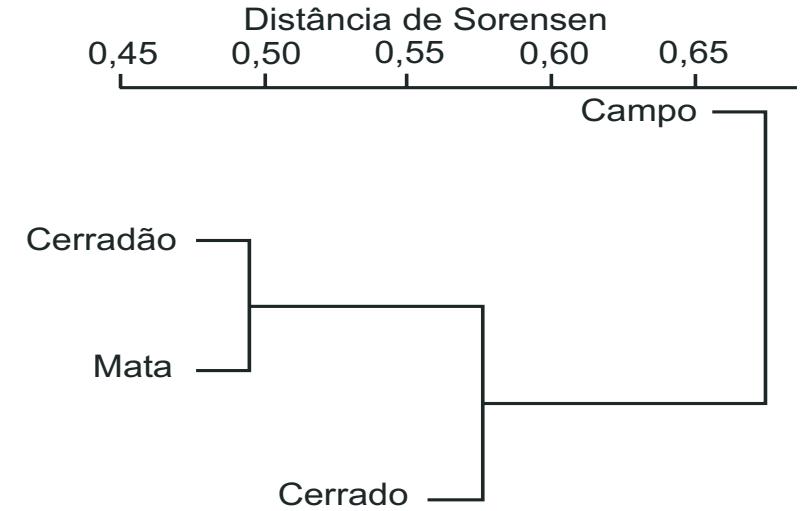

Figura 3. Dendrograma das quatro fitofisionomias de Cerrado amostradas no estudo gerado por UPGMA, utilizando a distância de Sorensen. A fitofisionomia mata refere-se à mata de galeria e cerrado indica cerrado sentido restrito.

ro de fitofisionomias nas localidades e a riqueza de plantas no Cerrado. Entretanto, não houve relação significativa entre o número de fitofisionomias e a riqueza de espécies de Arctiidae nas 14 localidades de Cerrado amostradas.

A relação entre a diversidade beta (distância de Sorensen) e a distância geográfica entre as localidades foi positiva e significativa, indicando que a similaridade diminui com o aumento da distância geográfica entre as localidades. A análise de UPGMA também confirma esse resultado, agrupando localidades próximas, tanto em São Paulo quanto em Goiás e Tocantins, as quais foram divididas em grupos do sudoeste e nordeste e do norte e sul, respectivamente.

Na separação do grupo Goiás (GO) e Pedregulho (SP) do restante das localidades, o fator distância pareceu não ser tão importante, pois localidades muito distantes entre si (mais de $800 \mathrm{~km}$ ), como Paranã e Chapadão do Céu, formaram grupos na análise de UPGMA. Sugere-se que o estado de preservação da vegetação seja o fator mais importante na separação desses dois grandes grupos, pois Goiás e Pedregulho apresentaram a vegetação mais alterada de todas as localidades amostradas.

Sugere-se que as diferenças na densidade da vegetação devam ser responsáveis pela separação dos grupos do Sudeste (Águas de Santa Bárbara, Angatuba, Assis, Luis Antônio, Porto Ferreira, Santa Rita do Passa Quatro e São Simão) e CentroOeste (Alto Paraíso de Goiás, Paranã, Caldas Novas e Chapadão do Céu). As localidades amostradas em SP eram compostas apenas por fitofisionomias florestais (cerradão e mata) e as localidades de GO e TO por fitofisionomias florestais, savânicas e campestres (mata, cerrado sentido restrito e campo sujo). Durigan et al. (2003), estudando as plantas arbóreas dos cerrados de SP, verificaram a existência de dois grandes grupos (áreas do leste e do oeste do Estado) e sugeriram que o fator que mais explicava a separação desses grupos era o tipo de fitofisionomia da vegetação (predominância de fitofisionomias florestais e de vegetação mais aberta). A análise de UPGMA para as fitofisionomias de Cerrado corrobora com os resultados de DuRIGAn et al. (2003), pois mostra que a fauna de Arctiidae coletada em fitofisionomias florestais (mata e cerradão) forma um grupo coeso, distinto daquele coletado nas fitofisionomias mais abertas (cerrado sentido restrito e campo sujo).

No geral, as localidades do grupo do Centro-Oeste apresentaram menores índices de similaridade entre si do que as do grupo do Sudeste. Os baixos índices de similaridade do grupo do Centro-Oeste refletem a alta heterogeneidade entre as localidades desse grande grupo (a amplitude nas distâncias geográficas entre as localidades e entre os tipos de fitofisionomias de cerrado foi bem maior neste grupo do que no grupo de SP).

Vários estudos em ambientes tropicais têm demonstrado que os Arctiidae podem ser favorecidos pela perturbação de habitats, podendo ser usado como grupo indicador de ambientes alterados (Willott 1999, Kitching et al. 2000, Summerville et al. 2004, Hilt \& FiedLer 2005, 2006). Isso geralmente é associado ao alto grau de polifagia das larvas de muitas espécies (HiLt \& FiedLer 2005, 2006). De fato, mais de $80 \%$ das espécies de larvas de Arctiidae coletadas no Cerrado são polífagas (MARQuis et al. 2002). Contudo, em nosso estudo, as localidades mais alteradas apresentaram baixa riqueza de espécies, não confirmando tal favorecimento.

A partir desse trabalho pode-se sugerir que a proximidade geográfica entre as localidades e as características locais da vegetação (tipo de fitofisioniomia e estado de preservação) explicam o padrão de diversidade beta encontrado para os Arctiidae coletados nas 14 localidades de Cerrado. Sabe-se, contudo, que a riqueza e a composição de espécies de Lepidoptera do Cerrado são relacionadas com uma série de outros fatores, principalmente o clima, altitude e solo (BROWN JR \& GIFFORD 2002). A relação da composição da fauna dessas mariposas com essas outras variáveis ambientais será objeto de estudos futuros. Apesar desse trabalho não ter concluído a existência de uma maior riqueza de arctiídeos em ambientes alterados, este grupo pode ser utilizado como indicador da qualidade dos habitats porque responde bem às alterações na vegetação. Além disso, esse trabalho demonstra que a alta heterogeneidade da vegetação do Cerrado observada por vários autores (ex. RATTER et al. 2003) pode ser estendida aos Arctiidae que utilizam as plantas como recurso. Por isso, é necessário que estudos sobre a composição local da fauna e flora sejam intensificados no Cerrado para assegurar que as futuras unidades de conservação protejam a maioria das espécies desse bioma.

\section{AGRADECIMENTOS}

A Vitor O. Becker pelo auxílio essencial nas identificações das mariposas e pelo empréstimo de bibliografia. Ao Dr. Adriano S. Melo pela ajuda fundamental em quase todas as coletas de campo, nas análises estatísticas e pela revisão do manuscrito. Ao Ibama, Agência Ambiental de Goiás e Instituto Florestal de São Paulo pelas licenças de coleta concedidas. Aos diretores, pesquisadores e funcionários das unidades de conservação visitadas pelo apoio logístico. A CAPES, CNPq, MMA (PROBIO) e ao PPG Ecologia UNB pelo apoio financeiro. 


\section{REFERÊNCIAS BIBLIOGRÁFICAS}

Brown JR, K.S. \& A.V.L. Freitas. 1999. Lepidoptera, p. 225-243. In: C.R.F. BRANDÃo \& E.M. Cancello (Eds). Biodiversidade do Estado de São Paulo: síntese do conhecimento ao final do século XX. São Paulo, Fapesp, vol. 5, 279p.

Brown JR, K.S. \& D.R GifFord. 2002. Lepidoptera in the cerrado landscape and the conservation of vegetation, soil and topographical mosaics, p. 201-217. In: P.S. Oliveira \& R.J. MARquis (Eds). The Cerrados of Brazil: Ecology and Natural History of a Neotropical Savanna. New York, Columbia University Press, 398p.

Brown Jr, K.S. \& O.H.H. MielKe. 1967. Lepidoptera of the Central Brazil Plateau. I. Preliminary list of Rhopalocera: Introduction, Nymphalidae, Libytheidae. Journal of Lepidopterists' Society 21 (2): 77-106.

Brown Jr, K.S. \& O.H.H. MielKe. 1968. Lepidoptera of the Central Brazil Plateau. III. Partial list for the Belo Horizonte area, showing the character of the southeastern "blend zone". Journal of Lepidopterists' Society 22 (3): 147-157.

CAmargo, A.J.A. 1999. Estudo comparativo sobre a composição e a diversidade de lepidópteros noturnos em cinco áreas da Região dos Cerrados. Revista Brasileira de Zoologia 16 (2): 369-380.

Camargo, A.J.A. \& V.O. Becker. 1999. Saturniidae (Lepidoptera) from the Brazilian Cerrado: composition and biogeographic relationships. Biotropica 31 (4): 696-705.

Colwell, R.K. \& J.A. Coddington. 1994. Estimating terrestrial biodiversity through extrapolation. Philosophical Transactions Royal Society of London, Series B, 345 (1311): 101-118.

Conservation International; Funatura; Fundação Biodiversitas \& Universidade de Brasília. 1999. Ações Prioritárias para a Conservação da Biodiversidade do Cerrado e Pantanal. Disponível na World Wide Web em: http://www.bdt.org.br/ workshop/cerrado/br/resultados [acesso em 04.III.2006].

DeVries, P.J.; D. Murray \& R. Lande. 1997. Species diversity in vertical, horizontal, and temporal dimensions of a fruitfeeding butterfly community in an Ecuadorian rainforest. Biological Journal of the Linnean Society 62 (3): 343-364.

DiAs, B.F.S. 1996. Cerrados: uma caracterização, p. 11-25. In: B.F.S. DiAs (Ed.). Alternativas de desenvolvimento dos Cerrados: manejo e conservação dos recursos naturais renováveis. Brasília, Funatura, 97p.

Durigan, G.; M.F. Siqueira; G.A.D.C. Franco; S. Bridgewater \& J.A. RAtTer. 2003. The vegetation of priority areas for cerrado conservation in São Paulo State, Brazil. Edinburgh Journal of Botany 60 (2): 217-241.

Heppner, J.B. 1991. Faunal regions and the diversity of Lepidoptera. Tropical Lepidoptera 2 (Suppl. 1): 1-85.

Hilt, N. \& K. FiedLer. 2005. Diversity and composition of Arctiidae moth ensembles along a successional gradient in the Ecuadorian Andes. Diversity and Distributions 11 (5): $387-$ 398.

Hitt, N. \& K. Fiedler. 2006. Arctiidae moth ensembles along a successional gradient in the Ecuadorian montane rain forest zone: how different are subfamilies and tribes? Journal of Biogeography 33 (1): 108-120.

Hilty, J. \& A. Merenlender. 2000. Faunal indicator taxa selection for monitoring ecossystem health. Biological Conservation 92 (2): 185-197.

Jacobson, N.L. \& S.J. Weller. 2002. A cladistic study of the Arctiidae (Lepidoptera) by using characters of immatures and adults. Lanham, Entomological Society of America, Thomas Say Publication in Entomology, Monographs, 98p.

Kitching, R.L.; A.G. OrR; L. Thalib; H. Mitchell; M.S. Hopkins \& A.W. Graham. 2000. Moths assemblages as indicators of environmental quality in remnants of upland Australian rain forest. Journal of Applied Ecology 37 (2): 284-297.

MAcEDo, R.H.F. 2002. The avifauna: ecology, biogeography, and behavior, p. 242-265. In: P.S. Oliveira \& R.J. MARQuis (Eds). The Cerrados of Brazil: Ecology and Natural History of a Neotropical Savanna. New York, Columbia University Press, 398p.

Mantovani, J.E. \& A. Pereira. 1998. Estimativa da integridade da cobertura vegetal do Cerrado/Pantanal através de dados TM/ LANDSAT. Ações prioritárias para conservação da biodiversidade do Cerrado e Pantanal. Brasília, Ministério do Meio Ambiente, Funatura, Conservational International, Fundação Biodiversitas, Universidade de Brasília. Disponível na World Wide Web em: http://www.bdt.org.br/workshop/ cerrado/br/inpe [acesso em 08.II.2006].

Marinho-Filho, J.; F.H.G. Rodriguez \& K.M. Juarez. 2002. The cerrado mammals: diversity, ecology, and natural history, p. 266284. In: P.S. Oliveira \& R.J. Marquis (Eds). The Cerrados of Brazil: Ecology and Natural History of a Neotropical Savanna. New York, Columbia University Press, 398p.

Marquis, R.J.; H.C. Morais \& I.R. Diniz. 2002. Interactions among cerrado plants and their herbivores: unique or typical? p. 306-328. In: P.S. Oliveira \& R.J. Marquis (Eds). The Cerrados of Brazil: Ecology and Natural History of a Neotropical Savanna. New York, Columbia University Press, 398p.

McCune, B. \& M.J. MefFord. 1997. Multivariate analysis of ecological data. Version 3.1. MjM Software, Gleneden Beach.

Mielke, O.H.H. 1968. Lepidoptera of the Central Brazil Plateau. II. New genera, species, and subspecies of Hesperiidae. Journal of Lepidopterist' Society 22 (1): 1-20.

Myers, N.; R.A. Mittermeier; C.G. Mittermeier; G.A.B. da Fonseca $\&$ J. Kent. 2000. Biodiversity hotspots for conservation priorities. Nature 403 (6772): 853-858.

Novotny, V. \& Y. BAsset. 2000. Rare species in communities of tropical insect herbivores: pondering the mystery of singletons. Oikos 89 (3): 564-572.

R Development Core Team. 2004. R: a language and environment for statistical computing. Vienna, R Foundation for Statistical Computing. Available in the World Wide Web at: http://www.R-project.org [accessed in 10.V.2006] 
Ratter, J.A.; J.F. Ribeiro \& S. Bridgewater. 1997. The Brazilian cerrado vegetation and threats to its biodiversity. Annals of Botany 80 (3): 223-230.

Ratter, J.A.; S. Bridgewater \& J.F. Ribeiro. 2003. Analysis of the floristic composition of the Brazilian cerrado vegetation III: comparation of the woody vegetation of 376 areas. Edinburgh Journal of Botany 60 (1): 57-109.

Ribeiro, J.F. \& B.M.T. Walter. 1998. Fitofisionomias do bioma Cerrado, p. 89-166. In: S.M. SANo \& S.P. Almeida (Eds). Cerrado: ambiente e flora. Planaltina, EMBRAPA-CPAC, 556p.

Robinson, G.S. \& K.R. TUCK. 1993. Diversity and faunistics of small moths (microlepidoptera) in Bornean rainforest. Ecological Entomology 18 (4): 385-393.
Scoble, M.J. 1995. The Lepidoptera: form, function and diversity. New York, Oxford University Press, 403p.

Summerville, K.S.; L.M. Ritter \& T.O. Crist. 2004. Forest moth taxa as indicators of lepidopteran richness and habitat disturbance: a preliminary assessment. Biological Conservation 116 (1): 9-18.

Willot, S.J.1999. The effects of selective logging on the distribution of moth in a Bornean rainforest. Philosophical Transactions of the Royal Society of London, Series B, 354 (1391): 1783-1790.

Recebido em 04.VII.2006; aceito em 30.VII.2007.

Apêndice I. Espécies de Arctiidae coletadas nas 14 localidades. A presença da espécie na localidade é indicada por X. As 21 espécies não identificadas não foram citadas. (1) Águas de Santa Bárbara, (2) Alto Paraíso de Goiás, (3) Angatuba, (4) Assis, (5) Caldas Novas, (6) Chapadão do Céu, (7) Goiás, (8) Luis Antônio, (9) Paranã, (10) Pedregulho, (11) Pirenópolis, (12) Porto Ferreira, (13) Santa Rita do Passa Quatro e (14) São Simão.

\begin{tabular}{|c|c|c|c|c|c|c|c|c|c|c|c|c|c|c|}
\hline Espécies & 1 & 2 & 3 & 4 & 5 & 6 & 7 & 8 & 9 & 10 & 11 & 12 & 13 & 14 \\
\hline \multicolumn{15}{|l|}{ Arctiinae } \\
\hline \multicolumn{15}{|l|}{ Arctiini } \\
\hline Hypercompe abdominalis (Walker, [1865]) & & & & & & & $x$ & & & & & & & \\
\hline Hypercompe cunigunda (Stoll, 1781) & & & & & & & & & & $x$ & & & & \\
\hline Hypercompe mus (Oberthür, 1881) & & & & & & & & & $x$ & & & & & \\
\hline Isia alcumena (Berg, 1882) & & $x$ & & & $x$ & & & & & & & & & \\
\hline Paracles aurantiaca (Rothschild, 1910) & & & & & & $\mathrm{x}$ & & & & & & & & \\
\hline Paracles phaeocera (Hampson, 1905) & & & & $x$ & $x$ & & & & & & & & $\mathrm{X}$ & \\
\hline Pseudalus limona Schaus, 1896 & & & $x$ & & $x$ & & & & $\mathrm{x}$ & $x$ & & & & \\
\hline Virbia medarda (Stoll, [1781]) & $x$ & & & $x$ & & & & & & & & & & \\
\hline Virbia subapicalis (Walker, 1854) & & & & & $x$ & & & & & & & & & $\mathrm{x}$ \\
\hline Virbia sp. & & & & & & $x$ & & & & & & & & \\
\hline \multicolumn{15}{|l|}{ Callimorphini } \\
\hline Utetheisa ornatrix (Linnaeus, 1758) & & & $x$ & $x$ & $x$ & $x$ & & & $\mathrm{x}$ & $x$ & $x$ & & $x$ & \\
\hline \multicolumn{15}{|l|}{ Ctenuchini } \\
\hline Aclytia heber (Cramer, 1780) & $x$ & $x$ & $x$ & $x$ & $\mathrm{x}$ & & $\mathrm{x}$ & $x$ & $x$ & $x$ & & $x$ & $x$ & $x$ \\
\hline Aclytia ventralis (Guérin, 1844) & & & $x$ & $x$ & & $\mathrm{x}$ & & & & & & $x$ & & \\
\hline Aclytia sp. & & $x$ & & & & $x$ & & & & & & & & \\
\hline Aethria haemorrhoidalis (Stoll, 1790) & & $x$ & & & & & & & & & & & & \\
\hline Argyroeides variegata Kaye, 1911 & & & $x$ & $x$ & & & & & & & & & & \\
\hline Chrysostola discoplaga Schaus, 1905 & & & & $x$ & & & & & & & & & & \\
\hline Chrysostola dycladioides (Heylaerts, 1890) & & & & & & & & & $x$ & & & & & \\
\hline Chrysostola nivaca (Jones, 1914) & $x$ & $x$ & & & $x$ & $x$ & & & $\mathrm{x}$ & $x$ & & & & \\
\hline Correbidia terminalis (Walker, 1856) & $x$ & & $x$ & & & & & & $x$ & & & & & \\
\hline Delphyre discalis (Druce, 1905) & & & & $x$ & $\mathrm{x}$ & & & $x$ & & & & $x$ & $x$ & $x$ \\
\hline Delphyre dizona (Druce, 1898) & & & $x$ & $x$ & & & & & & & $x$ & $x$ & & \\
\hline Delphyre hebes Walker, 1854 & & & $x$ & & & $x$ & & & & & & & & \\
\hline Delphyre pyroperas Hampson, 1911 & $x$ & & $x$ & $x$ & & & & & & & & & & \\
\hline
\end{tabular}


Apêndice I. Continuação.

\begin{tabular}{|c|c|c|c|c|c|c|c|c|c|c|c|c|c|c|}
\hline Espécies & 1 & 2 & 3 & 4 & 5 & 6 & 7 & 8 & 9 & 10 & 11 & 12 & 13 & 14 \\
\hline Episcepsis thetis (Linnaeus, 1771) & & & $x$ & & & & & & & & & & & \\
\hline Eucereon cineta Schaus, 1896 & & & & & & & & & & & & & & $\mathrm{x}$ \\
\hline Eucereon marcata Schaus, 1901 & & & & & $x$ & & & & & $x$ & & & & \\
\hline Eucereon obscura (Möschler, 1878) & & & & & & & & & & & $x$ & & & \\
\hline Eucereon rosa (Walker, 1854) & & & & & & & & & & & & $x$ & & \\
\hline Eucereon sylvius (Stoll, 1790) & & & & $x$ & $x$ & & & & & & & & & \\
\hline Eucereon sp. & & & & $x$ & & & & & & & & & & \\
\hline Eucereon sp.2 & & & & & $x$ & & & & & & & $x$ & & \\
\hline Euceriodes wernickei (Draudt, 1917) & & & & & & & $x$ & & & & & & & \\
\hline Euclera diversipennis (Walker, 1854) & & & & & & & & $x$ & & & & & & \\
\hline Euclera rubricincta (Burmeister, 1878) & & & & & & $x$ & & & & & & & & \\
\hline Euclera stretchii (Butler, 1876) & & & & & & & & & & & & & & $x$ \\
\hline Heliura rhodophila (Walker, 1856) & & & & $x$ & & & & & & $x$ & & & & \\
\hline Hyaleucerea phaeosoma Hampson, 1905 & & & & & & & & & & & & & & $x$ \\
\hline Napata leucosthelus Butler, 1876 & & & & & & & $x$ & & & & $x$ & & & \\
\hline Nelphe confinis (Herrich-Schaffer, [1855]) & & & $x$ & & & & & & & & & & & \\
\hline Philoros rubriceps (Walker, 1854) & & & & & $x$ & & $x$ & & & $x$ & & $x$ & $x$ & \\
\hline Pionia lycoides (Walker, 1854) & & & $x$ & & & & & & & & & & & \\
\hline Pionia nigrodorsalis (Draudt, 1915) & & & & & $x$ & & & & & & & & & \\
\hline Pseudaethria cessogae Schaus, 1924 & & & $x$ & & & & & & & & & & & \\
\hline Pseudohyaleucerea vulnerata (Butler, 1875) & $x$ & & & $x$ & & & & & & & & & & \\
\hline Pseudosphex aequalis (Walker, 1864) & & & & & & & $x$ & & $x$ & & & & & \\
\hline Pseudosphex sodalis Draudt, 1915 & & & & & & & & & $x$ & & & & $x$ & \\
\hline Tipulodes rubriceps Dognin, 1912 & & & & & & & $x$ & & & & & & & \\
\hline \multicolumn{15}{|l|}{ Euchromiini } \\
\hline Agerocha eone Hübner 1827 & & & & & & & & & & $x$ & & & & \\
\hline Cosmosoma achemon (Fabricius, 1781) & & & & & & & $x$ & & & & & & & \\
\hline Cosmosoma auge (Linnaeus, 1767) & & & & & & & & & & $x$ & & & & \\
\hline Cosmosoma centrale (Walker, 1854) & & & $x$ & & & & & & & & & & & \\
\hline Cosmosoma elegans Butler, 1876 & & & $x$ & & & & & & & & & & & \\
\hline Cosmosoma impar (Walker, 1854) & & & & & & & & & & & & & $x$ & \\
\hline Cosmosoma nigriscens Rothschild, 1911 & & & & & $x$ & & & & & & & & & \\
\hline Cosmosoma rasera Jones, 1914 & & & & & $x$ & & & & & & & & & \\
\hline Cosmosoma remotum (Walker, 1854) & & & $x$ & $x$ & & & & $x$ & & $x$ & & & $x$ & \\
\hline Cosmosoma restrictum Butler, 1876 & & $x$ & & $x$ & $x$ & & & & $x$ & $x$ & & & & $x$ \\
\hline Cosmosoma subflammans (Walker, 1856) & & & $x$ & & & & & & & & & & & \\
\hline Cosmosoma sp. & & & & & & $x$ & & & & & & & & \\
\hline Dycladia correbioides Felder, 1869 & & & $x$ & & & & & & & $x$ & & & & \\
\hline Dycladia lucetius (Stoll, 1781) & $x$ & $x$ & $x$ & $x$ & $x$ & $x$ & $x$ & $x$ & $x$ & $x$ & $x$ & & $x$ & \\
\hline Eurota histrio (Guérin, 1843) & & & & & $x$ & & & & & & & & & \\
\hline Eurota sp. & & $x$ & & & & & & & & & & & & \\
\hline Eurota sp.2 & & $x$ & & & & & & & & & & & & \\
\hline Eurota sp.3 & & $x$ & & & & & & & & & & & & \\
\hline Hyda basilutea (Walker, 1854) & & & $x$ & & $x$ & & & & & & & & & \\
\hline
\end{tabular}


Apêndice I. Continuação.

\begin{tabular}{|c|c|c|c|c|c|c|c|c|c|c|c|c|c|c|}
\hline Espécies & 1 & 2 & 3 & 4 & 5 & 6 & 7 & 8 & 9 & 10 & 11 & 12 & 13 & 14 \\
\hline Lepidoneiva erubescens (Butler 1876) & & $x$ & $\mathrm{x}$ & & $x$ & $x$ & & $\mathrm{x}$ & $x$ & & $x$ & $x$ & $x$ & $x$ \\
\hline Leucotmemis emergens (Walker, 1864) & & & & & $x$ & $x$ & & $x$ & & & & & & \\
\hline Loxophlebia leucothema Dyar, 1914 & & & & & & & & & $x$ & & & & $x$ & \\
\hline Loxophlebia peralta Schaus, 1912 & & & & & & & & $x$ & & & & & & \\
\hline Mesothen desperata (Walker, 1856) & & & & & & & & & & $x$ & & & & \\
\hline Mesothen pyrrhina Jones, 1914 & & & $x$ & & & & & & & & & & & $x$ \\
\hline Neotrichura nigripes (Heylaerts, 1890) & & & $x$ & & & & & & & & & & & \\
\hline Nyridela acroxantha (Perty, 1834) & & & $\mathrm{x}$ & & & & & & & & & & & \\
\hline Pheia albisigna (Walker, 1854) & & & $\mathrm{x}$ & & $x$ & $x$ & & & & & & & $x$ & \\
\hline Pheia haematosticta Jones, 1908 & & & & & $x$ & $x$ & & & & & & & & \\
\hline Pheia haemopera Schaus, 1898 & & & & & $x$ & $x$ & & & $x$ & & & & & \\
\hline Pheia picta (Walker, 1854) & & & $\mathrm{x}$ & & & & & & & & & & & \\
\hline Pheia seraphina (Herrich-Schaeffer, 1854) & $\mathrm{X}$ & $\mathrm{X}$ & $\mathrm{x}$ & & $x$ & $x$ & & & $X$ & & & & $\mathrm{X}$ & \\
\hline Phoenicoprocta baeri Rothschild, 1911 & & & & & $x$ & $x$ & & & $x$ & & & & & \\
\hline Phoenicoprocta haemorrhoidalis (Fabricius, 1775) & & & $\mathrm{x}$ & & & & & & & & & & & \\
\hline Phoenicoprocta ignicauda Draudt, 1915 & & & & & & $x$ & & & & & & & & \\
\hline Phoenicoprocta steinbachi Rothschild, 1911 & & & & & & & & & $x$ & & & & & \\
\hline Poliopastea indistincta (Butler, 1876) & & $\mathrm{X}$ & & & $\mathrm{X}$ & & & & & & & & & \\
\hline Pseudomya desperata (Walker, 1865) & & & & & & & & & & & & & & $x$ \\
\hline Pseudomya tipulina (Hübner, [1812]) & & & & & $\mathrm{x}$ & & & & & & & & & \\
\hline Saurita cassandra (Linnaeus, 1758) & & & & & & & $x$ & & & $\mathrm{x}$ & & & & \\
\hline Saurita sericea (Herrich-Schaffer, 1854) & & & & & $\mathrm{x}$ & & & & & & & & & \\
\hline Saurita triangulifera (Druce, 1898) & & & & & & & & & $x$ & & & & & \\
\hline Telioneura albapex (Druce, 1898) & & & & $x$ & & & & & & & & & & \\
\hline \multicolumn{15}{|l|}{ Pericopini } \\
\hline Dysschema boisduvalii (van der Hoeven e de Vriese, 1840) & & & & & & & & & & $\mathrm{X}$ & & & & \\
\hline Dysschema hypoxantha Hübner, 1818 & & & & & $\mathrm{X}$ & & & & & & & & & \\
\hline Dysschema lucifer (Butler, 1873) & & & $\mathrm{x}$ & & & & & & & & & & & \\
\hline Dysschema sacrifica (Hübner, [1831]) & & $\mathrm{X}$ & & & & & & & & & & & & \\
\hline \multicolumn{15}{|l|}{ Phaegopterini } \\
\hline Agaraea semivitrea Rothschild, 1909 & $\mathrm{x}$ & & $\mathrm{x}$ & $x$ & $\mathrm{x}$ & & & & & & & & $\mathrm{X}$ & \\
\hline Agaraea sp. & & & & & & $x$ & & & & & & & & \\
\hline Amaxia theon Druce, 1900 & & & & & $x$ & & & & & & & & & \\
\hline Aphyle cuneata Hampson, 1905 & & $\mathrm{X}$ & & & & & & & & & & & & \\
\hline Bertholdia almeidai Travassos, 1950 & & & $\mathrm{X}$ & & & & & & & & & & & \\
\hline Disconeura lutosa (Hübner, [1823]) & & & & & & & & & $x$ & & & & & \\
\hline Halysidota underwoodi Rothschild, 1909 & & & & & & & & $\mathrm{x}$ & & $\mathrm{x}$ & & & & $\mathrm{x}$ \\
\hline Hyperandra appendiculata (Herrich-Schäffer, [1856]) & $\mathrm{X}$ & & $\mathrm{x}$ & $x$ & $x$ & & & & & & & & & \\
\hline Hyperthaema hoffmannsi Rothschild, 1909 & & & $x$ & & & & & & & & & & & \\
\hline Hyperthaema ignifera Rawlins & & & $\mathrm{x}$ & & $x$ & & & & & & & & & \\
\hline Hyponerita similis Rothschild, 1909 & & & & & & & & & & & $x$ & & & \\
\hline Idalus carinosa (Schaus, 1905) & & & & & $x$ & $x$ & & & & & & & & \\
\hline Idalus dares Druce, 1894 & & & & & $x$ & & & & & & & & & \\
\hline Leucanopsis biedala (Schaus, 1941) & & & $\mathrm{x}$ & & & & & & & & & & & \\
\hline Leucanopsis lineata (Schaus, 1894) & & & & & & $X$ & & & & & & & & \\
\hline
\end{tabular}


Apêndice I. Continuação.

\begin{tabular}{|c|c|c|c|c|c|c|c|c|c|c|c|c|c|c|}
\hline Espécies & 1 & 2 & 3 & 4 & 5 & 6 & 7 & 8 & 9 & 10 & 11 & 12 & 13 & 14 \\
\hline Leucanopsis rosetta (Schaus, 1896) & & $\mathrm{x}$ & & & & & & & & & & & & \\
\hline Leucanopsis strigulosa (Walker, 1855) & & & $\mathrm{X}$ & & & & & & & & & & & \\
\hline Leucanopsis umbrosa (Hampson, 1901) & & & & & $\mathrm{X}$ & & & & & & & & & \\
\hline Leucanopsis sp. & & & & & & $x$ & & & & & & & & \\
\hline Lophocampa citrina (Sepp, [1852]) & $x$ & $x$ & $x$ & & $x$ & & $x$ & & & & & $x$ & & $x$ \\
\hline Lophocampa modesta (Kirby, 1892) & & & & & & & & & & & & & & $x$ \\
\hline Mazaeras francki (Schaus, 1899) & & & & & & & $x$ & & & & & & & \\
\hline Mazaeras janeira (Schaus, 1892) & & & & & & & & & & $\mathrm{x}$ & & & & \\
\hline Munona iridescens Schaus, 1894 & & & $\mathrm{X}$ & & & & & & & & & & $x$ & \\
\hline Neritos flavimargo Joicey \& Talbot, 1916 & & & & & & $x$ & & & & & & & & \\
\hline Opharus procroides Walker, 1855 & & & $x$ & & & & & & & & & & & \\
\hline Ormetica xanthia (Hampson, 1901) & & & & & $x$ & & & & & & & & & \\
\hline Pareuchaetes aurata (Butler, 1875) & & & & $x$ & & & & & & & & & & \\
\hline Parevia vulmaria Schaus, 1924 & & & $\mathrm{x}$ & & & & & & & & & & & \\
\hline Pelochyta lystra (Druce, 1884) & & & $\mathrm{x}$ & & & & & & & & & & & \\
\hline \multicolumn{15}{|l|}{ Lithosiinae } \\
\hline \multicolumn{15}{|l|}{ Cisthenini } \\
\hline Ripha mathildae (Köhler, 1924) & & & & & & & & & & $\mathrm{X}$ & & & & \\
\hline Ripha vivia (Watson, 1975) & & & & & & $X$ & & & & & & & & \\
\hline Symphlebia abdominalis (Herrich-Schäffer, [1855]) & $x$ & & $\mathrm{x}$ & $X$ & & & & & & $x$ & & & & \\
\hline Tessella sertata (Berg, 1882) & & & & & & & $x$ & & & & & & & \\
\hline Trichromia flexuosa (Schaus, 1911) & & & & & $\mathrm{X}$ & & & & & & & & & \\
\hline Trichromia sp. & & & & & & & & & & & $\mathrm{X}$ & & & \\
\hline Barsinella mirabilis Butler, 1878 & $x$ & $X$ & & $x$ & & $x$ & & $x$ & & & & & & \\
\hline Callisthenia plicata (Butler, 1877) & $x$ & $x$ & & $\mathrm{x}$ & & & & $x$ & & & & & & $x$ \\
\hline Cisthene discistriga (Dognin, 1912) & & & & $x$ & & $x$ & & & & & & $\mathrm{X}$ & & $x$ \\
\hline Cisthene pygmaea (Schaus, 1905) & & & & & & $x$ & & & & & & & & \\
\hline Cisthene rosaceae (Schaus, 1896) & $x$ & & $\mathrm{x}$ & & & & & & & & & & & \\
\hline Cisthene ruficollis (Schaus, 1896) & & & $x$ & & & & & & & & & & & \\
\hline Cisthene subrubra (Schaus, 1905) & & & & $X$ & $\mathrm{x}$ & & & $x$ & & $x$ & $X$ & & $\mathrm{X}$ & \\
\hline Cisthene triplaga Hampson, 1905 & $x$ & & $\mathrm{x}$ & $x$ & $x$ & & $\mathrm{X}$ & $x$ & & & & $x$ & $x$ & \\
\hline Cisthene xanthospila (Hampson, 1900) & & & & & $x$ & & & & & & $x$ & & & \\
\hline Cisthene sp. & & & & & & $x$ & & & & & & & & \\
\hline Cisthene sp.2 & & & $\mathrm{x}$ & & & & & & & & & $x$ & & \\
\hline Dolichesia subcoccinea (Schaus, 1905) & & & & $x$ & & & & & & & & & & \\
\hline Euthyone simplex (Walker, 1854) & $x$ & $x$ & $\mathrm{x}$ & $x$ & $x$ & $x$ & & $x$ & & & & $x$ & & $x$ \\
\hline Lycomorphodes correbioides Schaus, 1911 & & & $x$ & & & & & & & & & & & \\
\hline Lycomorphodes strigosa (Butler, 1877) & & & $\mathrm{x}$ & & & & & & & & & $x$ & & $x$ \\
\hline Odozana domina (Schaus, 1896) & $X$ & & $x$ & $x$ & & $x$ & & $x$ & & & $x$ & $x$ & $\mathrm{x}$ & $x$ \\
\hline Odozana obscura (Schaus, 1896) & $x$ & & $x$ & $x$ & & $x$ & & & & & & & & $x$ \\
\hline Prepiella miniola Hampson, 1900 & $x$ & & & $x$ & & $x$ & & & & & & & & \\
\hline Talara ditis (Butler, 1878) & & & $x$ & $x$ & & & & & & & & & & \\
\hline Talara grisea Schaus, 1896 & & $x$ & & & & & & & & & & & & \\
\hline Talara nigroplagiata Rothschild, 1913 & & & & & & & & $x$ & & & & & & \\
\hline Talara niveata Butler, 1878 & & & $\mathrm{X}$ & & & & & & & & & & & \\
\hline
\end{tabular}


Apêndice I. Continuação.

\begin{tabular}{|c|c|c|c|c|c|c|c|c|c|c|c|c|c|c|}
\hline Espécies & 1 & 2 & 3 & 4 & 5 & 6 & 7 & 8 & 9 & 10 & 11 & 12 & 13 & 14 \\
\hline Zonoda dives Schaus, 1896 & & & $x$ & $x$ & & & & & & & & $x$ & & \\
\hline Zonoda endoxantha (Hampson, 1903) & & & $x$ & & & & & & & & & & & \\
\hline \multicolumn{15}{|l|}{ Eudesmiini } \\
\hline Josiodes fallax Butler, 1877 & & & & & & & & & & & $\mathrm{x}$ & & & \\
\hline \multicolumn{15}{|l|}{ Lithosiini } \\
\hline Agylla involuta Hampson, 1900 & & & $\mathrm{X}$ & & & $x$ & & & & & & $x$ & & \\
\hline Agylla marcata (Schaus, 1894) & & & $x$ & & & & & & & & & & & \\
\hline Agylla polysemata Schaus, 1899 & & & $x$ & & & & & & & & & & & \\
\hline Agylla separata Schaus, 1894 & & & $x$ & & & & & & & & & & & \\
\hline Agylla sp.1 & & & $x$ & & & & & & & & & $x$ & & \\
\hline Agylla sp.2 & & & & & & $\mathrm{x}$ & & & & & & & & \\
\hline Agylla sp.3 & & & $\mathrm{x}$ & & & & & & & & & & & \\
\hline Agylla sp.4 & & & $\mathrm{x}$ & & & & & & & & & & & \\
\hline Apistosia judas Hübner, 1818 & & & & & & & & $x$ & & & & $x$ & $x$ & $x$ \\
\hline Balbura dorsisigna Walker, 1854 & & $x$ & & & & & & & & & & & & \\
\hline Epeiromulona hamata Field, 1952 & & & & & & $x$ & & & & $x$ & $x$ & & & \\
\hline Lamprostola hercyna (Draudt, 1885) & $\mathrm{x}$ & & $x$ & & & & & & & & & & & \\
\hline Lamprostola olivacea (Schaus, 1896) & & & & & & & & & & & & & & $x$ \\
\hline Lamprostola pascuala (Schaus, 1896) & $x$ & & $\mathrm{x}$ & & & & & & & & & & & \\
\hline Metalobosia invarda Schaus, 1905 & & & & & & & & & & & & & & $x$ \\
\hline Nodozana bellicula Schaus, 1905 & $x$ & & & & & & & & & & & & & \\
\hline Nodozana jucunda Jones, 1914 & $x$ & & $x$ & $x$ & $\mathrm{x}$ & $\mathrm{x}$ & & $\mathrm{x}$ & & & $x$ & $x$ & $x$ & \\
\hline Parablavia sadima (Schaus, 1896) & & & & & $x$ & $x$ & & & & & & & & \\
\hline Trichomelia celenna Schaus, 1892 & & & $x$ & & & & & & & & $x$ & & & \\
\hline
\end{tabular}

